\title{
The treatment of coronary artery aneurysm with a hybrid approach
}

\author{
Mehmet Acıpayam๑, Erdinç Eroğlu๑, Alptekin Yasım๑ \\ Department of Cardiovascular Surgey, Kahramanmaraş Sütçü İmam University School of Medicine, Kahramanmaraş, Turkey
}

\begin{abstract} treated with a hybrid approach, very rare case in the literature.

Toronary artery aneurysms (CAA) are reported incidence of $0.02-5.2 \%$ [1-4]. The most common etiological cause is atherosclerosis. Other causes are congenital factors, inflammation, connective tissue diseases, drug use, or procedural trauma $[1,2]$. Ischemic complications associated with thrombus and distal embolization are frequently observed in aneurysms. However, rupture is the most feared complication.
\end{abstract}

Objectives: Coronary artery aneurysms (CAAs) are rare cases that generally present with ischemic symptoms. Although coronary artery bypass surgery is widely performed in treatment, endovascular procedures have also begun being used in recent years. We describe a patient developing acute myocardial infarction due to CAA at a young age, despite the absence of any risk factor, followed by rapidly growing aneurysm. This patient was

Keywords: Coronary artery aneurysm, hybrid treatment, coil embolization, coronary artery bypass surgery
CAAs are generally treated surgically. However, endovascular procedures have also begun being used in recent years. Another option is medical treatment. However, a hybrid method consisting of surgery and endovascular procedures has not to date been used to treat this disease. We report the first case of CAA treated using a hybrid method. No risk factors were present in this young patient.

\section{CASE PRESENTATION}

An 18-year-old male patient presented due to chest pain. In coronary angiography, the proximal left anterior descending artery (LAD) was completely obstructed. Emergency percutaneous coronary intervention was attempted, but was unsuccessful. The patient was transferred to the coronary intensive care unit. It was planned to perform interventional treatment for total occlusion lesions at another session. After which patency was achieved in the LAD. Aneurysmatic dilation, $2.5 \mathrm{~cm}$ in diameter was observed in the proximal LAD, and stenosis was present after dilation (Fig. 1). A stent was implanted in the LAD. Two and a half months subsequently, the patient again presented with chest pain. Control angiography revealed that the $2.5 \mathrm{~cm}$ aneurysm had grown to $4.5 \mathrm{~cm}$ (Fig. 2), and the patient was referred for surgery. The presence of connective tissue disease was investigated, but no pathology was determined. There was no risk factor for atherosclerosis.

the aneurysm was very close to the bifurcation, we decided to adopt a hybrid approach in order to reduce the surgical risk. We decided to close the aneurysm using coils before performing coronary 


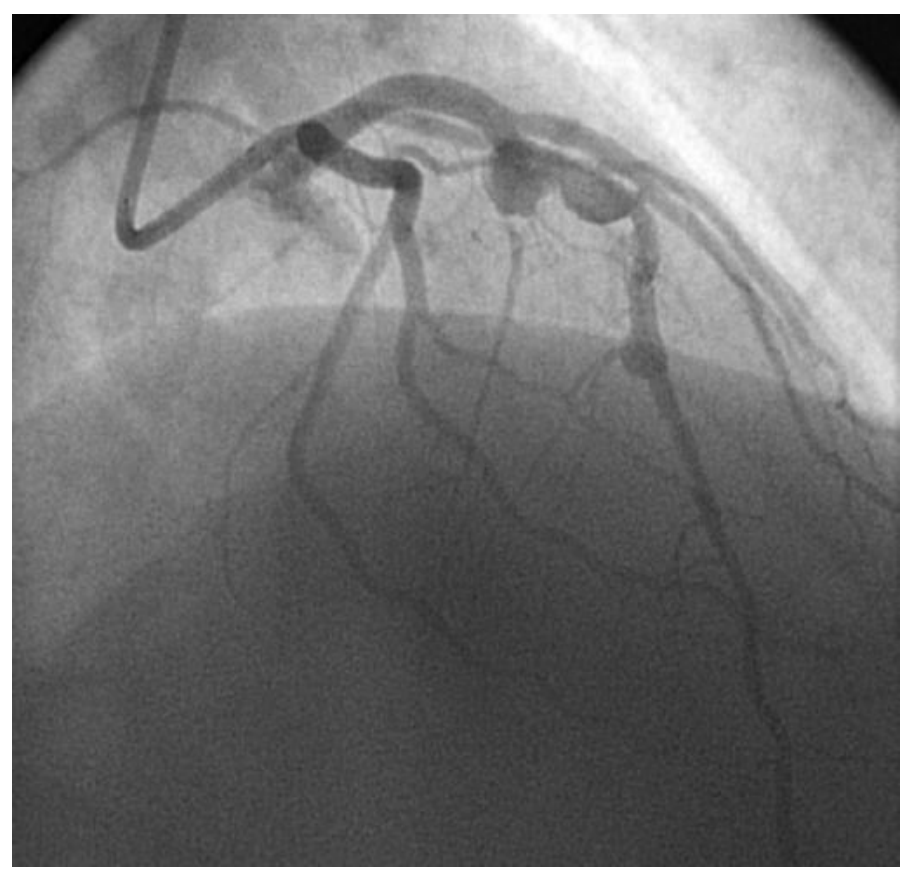

Fig. 1. First angiography showed multiple coronary aneurysms in LAD artery.

bypass. The proximal part of the aneurysm was first closed with a $4 \mathrm{~mm} \times 100 \mathrm{~mm}$ coil (Blockade Medical, Irvine, California, USA). However, the flow was not completely interrupted. A second $4 \mathrm{~mm} \times 100$ $\mathrm{mm}$ coil was inserted into the aneurysm, and no flow was observed at fluoroscopy. Cardiopulmonary bypass was established. The aneurysm in the LAD was visualized following medial displacement of the heart (Fig. 3). Cardiac arrest was established with a cross-

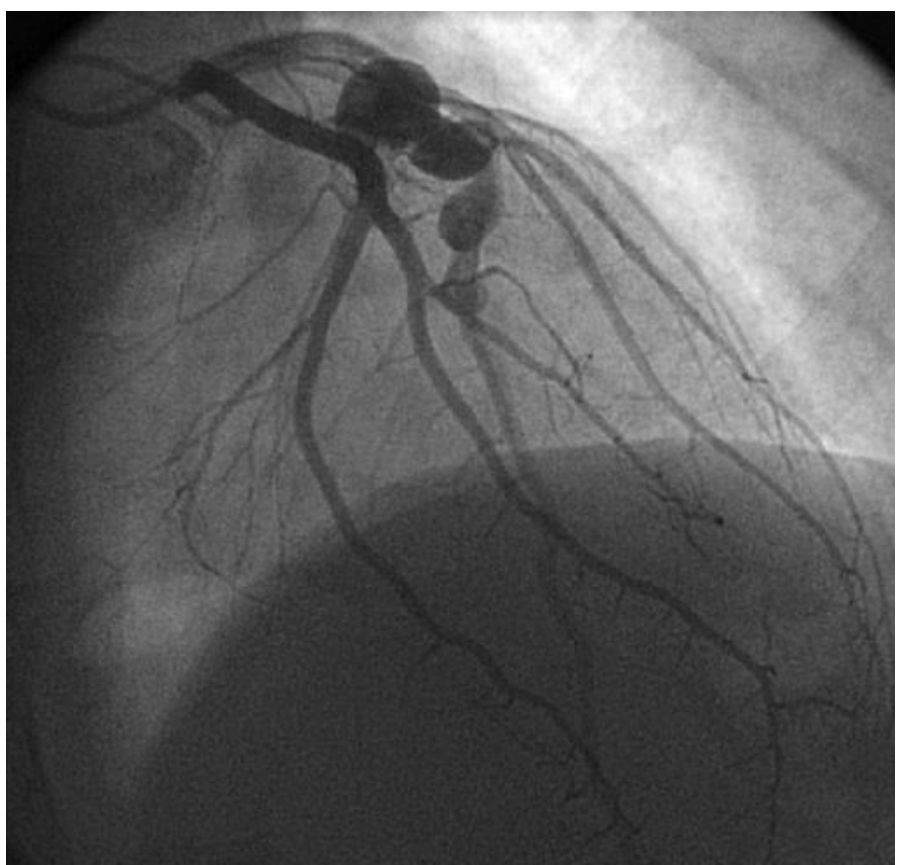

Fig. 2. After 2.5 months, control angiography showed enlargement of coronary artery aneurysms.

clamp to the aorta. The aneurysm sac was ligated from the distal part, and coronary artery bypass grafting was then performed on the LAD artery using the left anterior thoracic artery (LITA).

No problems developed in the postoperative period, and the patient remained in the ICU for one day. He was discharged on the fifth day postoperatively. Control coronary angiography was performed one month later. The LITA-LAD

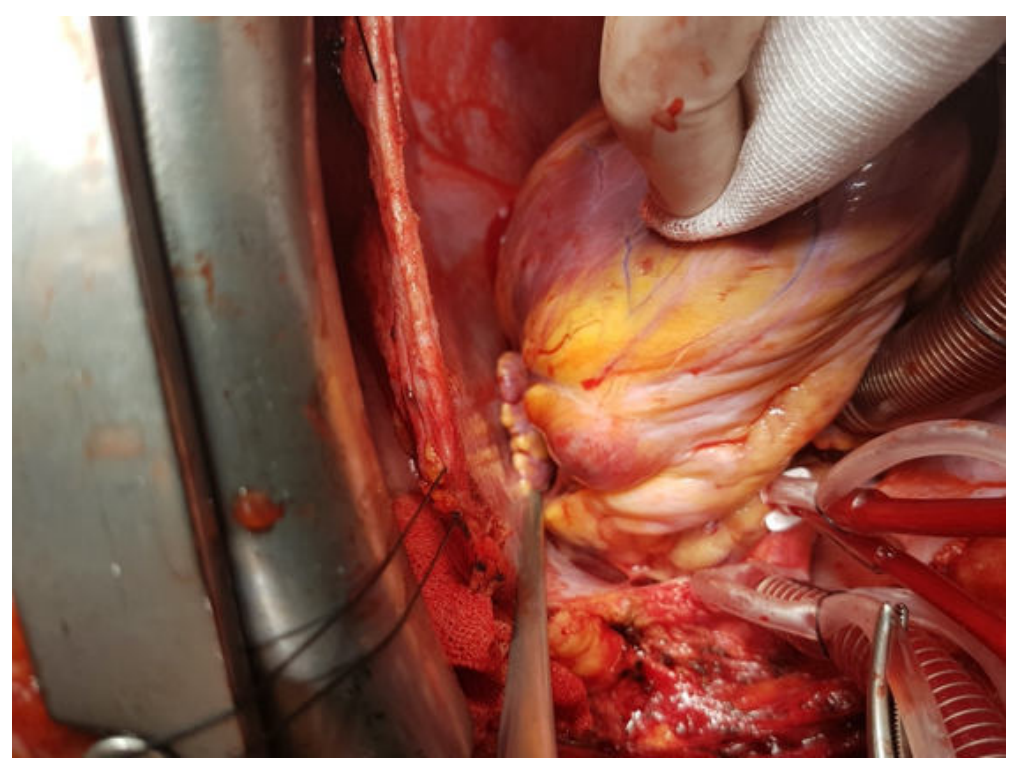

Fig. 3. Operative view of coronary artery aneurysm. 


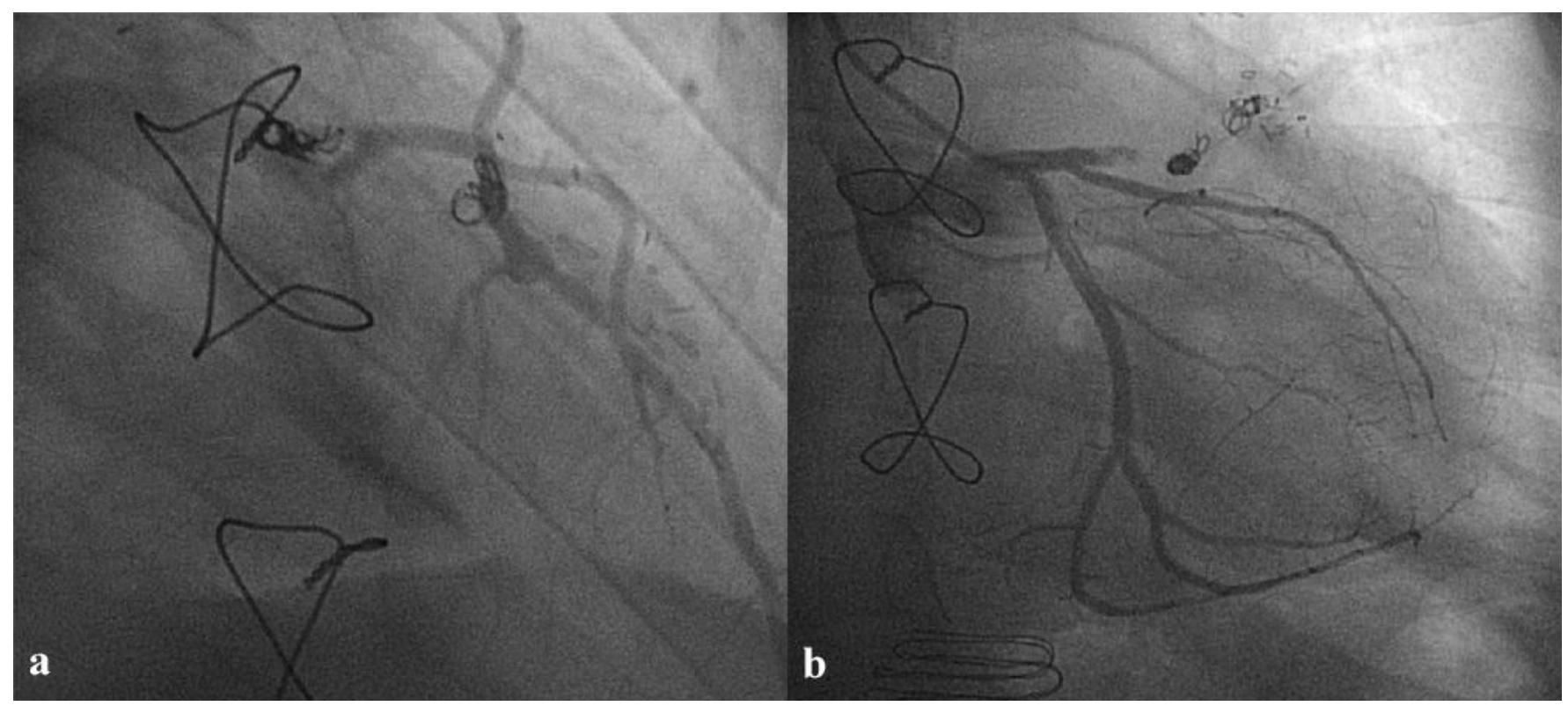

Fig. 4. Postoperative coronary angiography. (a) Patent LITA-LAD anastomosis and (b) the aneurysm sac could not be visualized.

anastomosis was patent (Fig. 4a) and the aneurysm sac could not be visualized (Fig. 4b).

\section{DISCUSSION}

Thrombotic complications are seen in more than $75 \%$ of CAAs $[2,3,5]$. Our patient also presented to hospital due to myocardial infarction. However, another fatal complication of CAAs is rupture. As in our case, rupture is inevitable if an aneurysm doubles in size in two months.

There is no consensus regarding the treatment of CAAs. Therapeutic options include surgery, percutaneous interventions, and medical treatment. Keyser et al. [3] stated that three out of five cases of aneurysms exceeding $5 \mathrm{~cm}$ in size died under conservative therapy. Surgery is the most common method in the treatment of CAAs. The most common operative technique is conventional coronary bypass surgery with ligation of the aneurysm. Other surgical methods include aneurysm resection and interposition graft, aneurysmorrhaphy, or patch plasty.

Endovascular therapy with stent-grafts has also been reported in CAA treatment in recent years. However, these grafts have high rates of stenosis and obstruction [1]. Surgery is therefore still regarded as the best option. The simplest technique in operative therapy is coronary artery bypass grafting with ligation of the distal and proximal aneurysm sac. However, if the aneurysm is very close to the left main artery, as in our case, ligating the proximal sac involves a risk of complication. Closing the proximal part with endovascular methods will be a good method in order to avoid complication. We therefore closed the beginning of the aneurysm using a coil, and surgery consisted of a simple, single coronary artery bypass operation. We know of no other case of CAA being treated with this method.

\section{CONCLUSION}

In conclusion, although there is still no consensus on the optimal treatment of CAAs, hybrid methods are an easy and safe option.

\section{Informed consent}

Written informed consent was obtained from the patient for publication of this case and any accompanying images.

\section{Conflict of interest}

The authors declared that there are no potential conflicts of interest with respect to the research, authorship, and/or publication of this article. 


\section{REFERENCES}

1. Cohen P, O'Gara PT. Coronary artery aneurysms: a review of the natural history, pathophysiology and management. Cardiol Rev 2008;16:301-4.

2. Beckmann E, Rustum S, Marquardt S, Merz C, Shrestha M, Martens A, et al. Surgical treatment of coronary artery aneurysms. J Card Surg 2017;32:674-9.

3. Keyser A, Hilker MK, Husser O, Diez C, Schmid C. Giant coronary aneurysms exceeding $5 \mathrm{~cm}$ in size. Interact Cardiovasc
Thorac Surg 2012;15:33-6.

4. Yavuz S, Eris C, Surer S, Toktas F. eComment. Coronary artery dilatation: ectasia or aneurysm. Interact Cardiovasc Thorac Surg 2013;17:636-7.

5. Crawley PD, Mahlow WJ, Huntsinger DR, Afiniwala S, Wortham DC. Giant coronary artery aneurysms: review and update. Tex Heart Inst J 2014;41:603-8. 